\title{
DIOPHANTINE INEQUALITIES IN FUNCTION FIELDS
}

\author{
CRAIG V. SPENCER
}

\begin{abstract}
This paper develops the Bentkus-Götze-Freeman variant of the DavenportHeilbronn method for function fields in order to count $\mathbb{F}_{q}[t]$-solutions to diagonal Diophantine inequalities in $\mathbb{F}_{q}((1 / t))$.
\end{abstract}

\section{INTRODUCTION}

Over 60 years ago, the Davenport-Heilbronn method (see [2]) was introduced to study non-trivial integral solutions of Diophantine inequalities. Let $k$ and $s$ be positive integers with $k>1$, and let $\tau$ be some fixed positive real number. Suppose that $\lambda_{1}, \ldots, \lambda_{s}$ are nonzero real numbers, not all in rational ratio. Let $N_{0}(P, \boldsymbol{\lambda})$ denote the number of solutions $\mathbf{x} \in[-P, P]^{s} \cap \mathbb{Z}^{s}$ that satisfy

$$
\left|\lambda_{1} x_{1}^{k}+\cdots+\lambda_{s} x_{s}^{k}\right|<\tau .
$$

Plainly, in the case that $k$ is an even number, we must impose the restriction that the numbers $\lambda_{i}$ do not all share the same sign in order to guarantee the existence of a nontrivial solution of $\lambda_{1} z_{1}^{k}+\cdots+\lambda_{s} z_{s}^{k}=0$ in $\mathbb{R}^{s}$. In [2], Davenport and Heilbronn proved that if $s>2^{k}$, then $N_{0}\left(P_{n}, \boldsymbol{\lambda}\right) \gg P_{n}^{s-k}$ for a sequence $\left(P_{n}\right)_{n=1}^{\infty}$ which increases to infinity. This sequence is determined from the convergents of the continued fraction expansion for an irrational number of the form $\lambda_{i} / \lambda_{j}$, and as a result, the sequence $\left(P_{n}\right)_{n=1}^{\infty}$ may be arbitrarily sparse. In the last decade, the Bentkus-Götze-Freeman version of the Davenport-Heilbronn method (see [1], [5], [6], and [14]) has been used to establish an asymptotic formula for $N_{0}(P, \boldsymbol{\lambda})$, valid for all large enough values of $P$, provided that

$$
s \geq k^{2}(\log k+\log \log k+O(1)),
$$

and an asymptotic lower bound for $N_{0}(P, \boldsymbol{\lambda})$, valid for all large enough values of $P$, provided that

$$
s \geq k(\log k+\log \log k+2+o(1)) .
$$

In this paper, we use the Bentkus-Götze-Freeman version of the Davenport-Heilbronn method to study the analogous problem in function fields.

In order to state our main result, it is first necessary to record some notation. Let $\mathbb{A}=\mathbb{F}_{q}[t]$ denote the ring of polynomials over $\mathbb{F}_{q}$, the finite field of $q$ elements. Let

Date: November 5, 2008.

2000 Mathematics Subject Classification. 11D75, 11P55, $11 \mathrm{~T} 55$.

Key words and phrases. Diophantine inequalities, Davenport-Heilbronn method, Hardy-Littlewood circle method, function fields.

This research was supported in part by NSF grants DMS-0601367 and DMS-0635607. 
$\mathbb{K}_{\infty}=\mathbb{F}_{q}((1 / t))$ be the completion of $\mathbb{K}=\mathbb{F}_{q}(t)$ at the infinite place. In this paper, we wish to exploit the basic observation that $\mathbb{A}$ behaves like $\mathbb{Z}$, that $\mathbb{K}$ behaves like $\mathbb{Q}$, and that $\mathbb{K}_{\infty}$ behaves like $\mathbb{R}$.

Let $k$ and $s$ be positive integers with $k>1$. Let $p$ denote the characteristic of $\mathbb{F}_{q}$. Each non-zero element $\alpha$ in $\mathbb{K}_{\infty}$ can be written as $\alpha=\sum_{i<n} a_{i} t^{i}$, where each $a_{i}$ is an element in $\mathbb{F}_{q}$ and $a_{n} \neq 0$. We define ord $\alpha$ to be $n$ and lead $(\alpha)$ to be $a_{n}$ in this situation. Furthermore, we define res $\alpha$ to be the coefficient of $t^{-1}$ in such an expansion, and we adopt the convention that ord $0=-\infty$. There exists a natural non-Archimedean valuation $\langle x\rangle=q^{\text {ord } x}$ on $\mathbb{K}_{\infty}$. For any real number $u$, we will let $\widehat{u}$ denote $q^{u}$. For a positive number $x$, we let $\log x=\max (1, \log x)$. When $k$ has a base- $p$ expansion $k=a_{0}+a_{1} p+\cdots+a_{n} p^{n}$ with $0 \leq a_{i} \leq p-1(0 \leq i \leq n)$, we define $\gamma(k)=\gamma_{q}(k)$ by

$$
\gamma(k)=a_{0}+a_{1}+\cdots+a_{n} .
$$

Define the constant $B=B_{q}(k)$ by

$$
B_{q}(k)= \begin{cases}1, & \text { when } k \leq 2^{\gamma-2} \\ \left(1-2^{-\gamma(k)}\right)^{-1}, & \text { when } k>2^{\gamma-2}\end{cases}
$$

Let

$$
s_{q, k}=B k(\log k+\log \log k+2+B \log \log k / \log k) .
$$

We are now in a position to state the main result of this paper.

Theorem 1.1. There exists a positive absolute constant $C$ with the following property. Suppose that $k$ and $s$ are natural numbers with $k>1$,

$$
s \geq s_{q, k}+C k \sqrt{\log \log k} / \log k
$$

and $\operatorname{char}\left(\mathbb{F}_{q}\right) \nmid k$. Let $\tau$ be some fixed integer, and let $\lambda_{1}, \ldots, \lambda_{s}$ be fixed non-zero elements of $\mathbb{K}_{\infty}$, not all in $\mathbb{F}_{q}(t)$-rational ratio. Suppose also that the equation

$$
\lambda_{1} z_{1}^{k}+\cdots+\lambda_{s} z_{s}^{k}=0
$$

has a non-trivial solution $\mathbf{z}$ in $\mathbb{K}_{\infty}^{s}$. Then, for all sufficiently large positive real numbers $P$, the number of $\mathbb{F}_{q}[t]$-solutions $N(P, \boldsymbol{\lambda})$ of

$$
\left\langle\lambda_{1} x_{1}^{k}+\cdots+\lambda_{s} x_{s}^{k}\right\rangle<\widehat{\tau}
$$

with $\left\langle x_{i}\right\rangle \leq \widehat{P}(1 \leq i \leq s)$, satisfies

$$
N(P, \boldsymbol{\lambda}) \gg \widehat{P}^{s-k}
$$

In (1.2), the implicit constant may depend on $s, k, q, \boldsymbol{\lambda}$, and $\tau$.

A few comments about the above theorem are in order. If $p \nmid k$, then $\gamma_{q}(k) \geq 2$, and it follows that $B_{q}(k)$ satisfies $1 \leq B_{q}(k) \leq 4 / 3$. Also, one should note that our function $s_{q, k}$ corresponds to the quantity $\widehat{G}_{q}(k)$ defined in [10] in the context of Waring's problem in function fields, and our work depends on mean value estimates arising from the use of efficient differencing technology for the latter problem. By incorporating any improvements to this machinery into the arguments of this paper, one would be able to get comparable improvements in Theorem 1.1. In the case that $k<\operatorname{char}\left(\mathbb{F}_{q}\right)$, a combination of Proposition 13 of [9], the amplification method discussed in Section 1 of [14], and the 
ideas in this paper would give a result similar to Theorem 1.1 with $s \geq 2^{k}+1$. This bound would be stronger than that of Theorem 1.1 for small values of $k$. Also, by Proposition 6.1, the equation $\lambda_{1} z_{1}^{k}+\cdots+\lambda_{s} z_{s}^{k}=0$ has a non-trivial solution $\mathbf{z} \in \mathbb{K}_{\infty}^{s}$ whenever $s \geq k^{2}+1$, whenever $q>k^{4}$ and $s \geq 2 k+1$, or whenever $(k, q-1)=1$ and $s \geq k+1$. Lastly, it is worth noting that the question of finding solutions to (1.1), where each $x_{i}$ is a monic, irreducible polynomial in $\mathbb{F}_{q}[t]$, has already been studied by Hsu in [7] through the use of the Davenport-Heilbronn method. Hsu's paper was restricted to the case that $k<p$ and

$$
s \geq \begin{cases}2^{k}+1, & \text { when } 2 \leq k<11, \\ 2\left[2 k^{2} \log k+k^{2} \log \log k+2 k^{2}-2 k\right]+1, & \text { when } k \geq 11\end{cases}
$$

The author would like to thank his advisor, Trevor Wooley, for many helpful conversations about this problem. Also, the author is grateful to Yu-Ru Liu and Trevor Wooley for copies of the preprints that inspired this work. Part of this paper was written during a visit to the University of Bristol, and the author would like to thank the Department of Mathematics for their generous hospitality and travel funding. The author also would like to thank the referee for providing valuable suggestions and corrections.

\section{The DAvenport-HeILBRonn METhod FOR FUnCtion FIELDS}

In this section, we set up the Davenport-Heilbronn Method for function fields. We will combine Hsu's version of the Davenport-Heilbronn method (see [7]) with the ideas of Bentkus and Götze (see [1]) and those of Freeman (see [5] and [6]) in order to prove Theorem 1.1.

Define a non-trivial additive character $e_{q}: \mathbb{F}_{q} \rightarrow \mathbb{C}^{\times}$by $e_{q}(a)=e^{2 \pi i \operatorname{tr}(a) / p}$, where $\operatorname{tr}: \mathbb{F}_{q} \rightarrow \mathbb{F}_{p}$ denotes the trace map. This character induces a map $e: \mathbb{K}_{\infty} \rightarrow \mathbb{C}^{\times}$ defined by $e(\alpha)=e_{q}($ res $\alpha)$. Let $\mathbb{T}$ be the compact additive subgroup of $\mathbb{K}_{\infty}$ given by $\mathbb{T}=\left\{\alpha \in \mathbb{K}_{\infty}:\langle\alpha\rangle<1\right\}$, and normalize a Haar measure $d \alpha$ on $\mathbb{K}_{\infty}$ so that $\int_{\mathbb{T}} d \alpha=1$. Note that this measure is translation-invariant and that for any integer $r$, we have

$$
\int_{\langle\alpha\rangle<\widehat{r}} d \alpha=\widehat{r}
$$

By Lemma 2.2 of [7], for $\tau \in \mathbb{Z}$, if we define a function $\chi_{\tau}: \mathbb{K}_{\infty} \rightarrow \mathbb{R}$ by

$$
\chi_{\tau}(\alpha)= \begin{cases}\widehat{\tau}, & \text { when }\langle\alpha\rangle<\widehat{\tau}^{-1}, \\ 0, & \text { when }\langle\alpha\rangle \geq \widehat{\tau}^{-1},\end{cases}
$$

we obtain a method of detecting when $\langle\beta\rangle<\widehat{\tau}$ for an element $\beta \in \mathbb{K}_{\infty}$ by noting that

$$
\int_{\mathbb{K}_{\infty}} e(\alpha \beta) \chi_{\tau}(\alpha) d \alpha= \begin{cases}1, & \text { when }\langle\beta\rangle<\widehat{\tau} \\ 0, & \text { when }\langle\beta\rangle \geq \widehat{\tau} .\end{cases}
$$

When $R$ and $P$ are positive numbers with $R \leq P$, we define the set of $R$-smooth polynomials $\mathcal{A}(P, R)$ to be the set of all $x \in \mathbb{A}$ satisfying both $\langle x\rangle \leq \widehat{P}$ and the property that whenever $\varpi \mid x$ for an irreducible polynomial $\varpi$, then $\langle\varpi\rangle \leq \widehat{R}$. We now can define our classical Weyl 
sum

$$
F(\alpha)=F(\alpha, P)=\sum_{\langle x\rangle \leq \widehat{P}} e\left(\alpha x^{k}\right)
$$

and our smooth Weyl sum

$$
f(\alpha)=f(\alpha, P, R)=\sum_{x \in \mathcal{A}(P, R)} e\left(\alpha x^{k}\right) .
$$

Let $F_{i}(\alpha)=F\left(\lambda_{i} \alpha\right)$ for $1 \leq i \leq s$, and let $f_{j}(\alpha)=f\left(\lambda_{j} \alpha\right)$ for $3 \leq j \leq s$. It now follows from (2.1) that the integral

$$
\int_{\mathbb{K}_{\infty}} F_{1}(\alpha) F_{2}(\alpha) f_{3}(\alpha) \cdots f_{s}(\alpha) \chi_{\tau}(\alpha) d \alpha
$$

counts the number of solutions $\mathbf{x} \in \mathbb{A}^{s}$ of

$$
\left\langle\lambda_{1} x_{1}^{k}+\cdots+\lambda_{s} x_{s}^{k}\right\rangle<\widehat{\tau}
$$

with $\left\langle x_{i}\right\rangle \leq \widehat{P}(i=1,2)$ and $x_{j} \in \mathcal{A}(P, R)(3 \leq j \leq s)$. Thus, the integral in (2.2) serves as a lower bound for $N(P, \boldsymbol{\lambda})$. For the remainder of this paper, whenever $R$ appears in a statement, implicitly or explicitly, we are asserting that there exists a positive number $\eta_{0}$ such that the statement holds whenever $R=\eta P$, where $0<\eta \leq \eta_{0}$.

It is worth noting that in the setting of diagonal Diophantine inequalities with solutions in $\mathbb{Z}^{s}$ (see [14]), one may use $s$ smooth Weyl sums instead of $s-2$ smooth Weyl sums and two classical Weyl sums. Were one to use only smooth Weyl sums with the approach in this paper, the main challenge arises when proving a result similar to Lemma 3.1 for smooth Weyl sums. The necessary minor arc bound for $f(\alpha)$ is available (see [10, Corollary $13.3]$ ), and one can implement the ideas of [12, Section 7] to obtain a major arc estimate for $f(\alpha)$ that is useful off of a slim set of the major arcs. However, the lack of function field analogues of various sieve bounds due to Fouvry and Tenenbaum in [4] is an impediment to proving an estimate for smooth Weyl sums on a very narrow set of the major arcs along the lines of [12, Lemma 8.5]. Were one to overcome this difficulty and prove an analogue of Lemma 3.1 for smooth Weyl sums, it would lead to a savings of one or two variables in Theorem 1.1.

Let $\mathfrak{n}$ denote the set of elements $\alpha$ of $\mathbb{T}$ satisfying the property that whenever $a$ and $g$ are elements of $\mathbb{A}$ such that $\langle g \alpha-a\rangle<\widehat{P}^{1-k}$ and $g \neq 0$, then $\langle g\rangle>\widehat{P}$. We say that a positive number $u>2 k-2$ is accessible to the exponent $k$ when there exists a positive number $\delta$ for which

$$
\int_{\mathfrak{n}}\left|F(\alpha, P)^{2} f(\alpha, P, R)^{u}\right| d \alpha \ll \widehat{P}^{u+2-k-\delta} .
$$

By Theorem 9.4, Corollary 13.3, and Lemma 14.1 of [10], it follows that there exists a positive absolute constant $C$ such that if

$$
u+5 \geq s_{q, k}+C k \sqrt{\log \log k} / \log k,
$$

then $u$ is accessible to the exponent $k$. Hence, Theorem 1.1 is a consequence of the following result. 
Theorem 2.1. Suppose that $k$ and $s$ are natural numbers with $k>1$ and char $\left(\mathbb{F}_{q}\right) \nmid k$. Furthermore, assume that $u>2 k-2$ is accessible to the exponent $k$ and that $s \geq u+5$. Let $\tau$ be some fixed integer, and let $\lambda_{1}, \ldots, \lambda_{s}$ be fixed non-zero elements of $\mathbb{K}_{\infty}$, not all in $\mathbb{F}_{q}(t)$-rational ratio. Suppose also that the equation

$$
\lambda_{1} z_{1}^{k}+\cdots+\lambda_{s} z_{s}^{k}=0
$$

has a non-trivial solution $\mathbf{z}$ in $\mathbb{K}_{\infty}^{s}$. Then, for all sufficiently large positive real numbers $P$, the number of $\mathbb{F}_{q}[t]$-solutions $N(P, \boldsymbol{\lambda})$ of

$$
\left\langle\lambda_{1} x_{1}^{k}+\cdots+\lambda_{s} x_{s}^{k}\right\rangle<\widehat{\tau}
$$

with $\left\langle x_{i}\right\rangle \leq \widehat{P}(1 \leq i \leq s)$, satisfies

$$
N(P, \boldsymbol{\lambda}) \gg \widehat{P}^{s-k}
$$

In (2.5), the implicit constant may depend on $s, k, q, \boldsymbol{\lambda}$, and $\tau$.

With the exception of Section 6, which will be used to investigate the solvability of (2.3) in $\mathbb{K}_{\infty}$, the remainder of this paper will be devoted to proving Theorem 2.1. In order to analyze the integral in $(2.2)$, we split up the subset of $\mathbb{K}_{\infty}$ for which the integrand is non-zero into two parts. Let

$$
S_{1}(P)=(\log \widehat{P})^{1 / 8}
$$

Define the major arc by

$$
\mathfrak{M}=\left\{\alpha \in \mathbb{K}_{\infty}:\langle\alpha\rangle<S_{1}(P) \widehat{P}^{-k}\right\}
$$

and the minor arc by

$$
\mathfrak{m}=\left\{\alpha \in \mathbb{K}_{\infty}: S_{1}(P) \widehat{P}^{-k} \leq\langle\alpha\rangle<\widehat{\tau}^{-1}\right\} .
$$

Theorem 2.1 is proved by demonstrating that for large enough values of $P$, one has

$$
\int_{\mathfrak{m}} F_{1}(\alpha) F_{2}(\alpha) f_{3}(\alpha) \cdots f_{s}(\alpha) \chi_{\tau}(\alpha) d \alpha=o\left(\widehat{P}^{s-k}\right)
$$

and

$$
\int_{\mathfrak{M}} F_{1}(\alpha) F_{2}(\alpha) f_{3}(\alpha) \cdots f_{s}(\alpha) \chi_{\tau}(\alpha) d \alpha \gg \widehat{P}^{s-k}
$$

We prove (2.6) in Section 4 (see Lemma 4.2) by combining mean value estimates with a Weyl-type estimate. The mean value estimates depend on the efficient differencing arguments in [10], and the Weyl-type estimate proved in Section 3 stems from the ideas of Bentkus, Götze, and Freeman in [1], [5], and [6]. We prove (2.7) in Section 5 (see Lemma $5.3)$ by using a line of attack similar to that of [9] and [10].

By multiplying each side of (2.4) by $\left\langle t^{-j}\right\rangle$ for some sufficiently large integer $j$, we may assume that $\widehat{\tau}<1$ and $0<\left\langle\lambda_{i}\right\rangle<1$ for $1 \leq i \leq s$. Since $\lambda_{1}, \ldots, \lambda_{s}$ are not all in $\mathbb{F}_{q}(t)$ rational ratio, there is no loss of generality in supposing that $\lambda_{2} / \lambda_{1} \notin \mathbb{K}$. Also, implicit constants in this paper may depend on $q, k, \boldsymbol{\lambda}, s, u, v$, and $\tau$. 


\section{A Weyl-type estimate}

In this section, we mimic the work in Section 2 of [14] to show that when $\alpha \in \mathfrak{m}$, one has $\left|F_{1}(\alpha) F_{2}(\alpha)\right|=o\left(\widehat{P}^{2}\right)$. Recall that $\mathfrak{n}$ denotes the set of elements $\alpha$ of $\mathbb{T}$ satisfying the property that whenever $a$ and $g$ are elements of $\mathbb{A}$ such that $\langle g \alpha-a\rangle<\widehat{P}^{1-k}$ and $g \neq 0$, then $\langle g\rangle>\widehat{P}$. It follows from [11] that there exists a small positive constant $\nu=\nu(q, k)$ such that

$$
\sup _{\alpha \in \mathfrak{n}}|F(\alpha)| \ll \widehat{P}^{1-\nu}
$$

Since one cannot use Weyl differencing to obtain such a bound without placing restrictions on the characteristic of $\mathbb{F}_{q}[t]$, this minor arc estimate is obtained via a combination of Vinogradov's mean value theorem with large sieve technology. It is worth noting that an asymptotic formula for $N(P, \boldsymbol{\lambda})$ would follow from a combination of the results of [11] with the ideas of this paper provided that $s$ is sufficiently large in terms of $k$. Since solutions of (2.4) are perfectly detected via (2.1), some of the technical difficulties that arise when studying the analogous problem in $\mathbb{Z}$ can be avoided.

Our first lemma demonstrates that good Diophantine approximations are produced by large Weyl sums.

Lemma 3.1. There is a positive constant $c$, depending at most on $k$ and $q$, with the following property. Suppose that $P$ is a real number, sufficiently large in terms of $k$ and $q$, and suppose that $\delta$ is a positive number with $\widehat{P}^{-\nu / 2} \leq \delta \leq 1$. Then, whenever $|F(\alpha)| \geq \delta \widehat{P}$, there exist $a$ and $g$ in $\mathbb{A}$ such that $(a, g)=1,1 \leq\langle g\rangle \leq c \delta^{-k}$, and $\langle g \alpha-a\rangle \leq c \delta^{-k} \widehat{P}^{-k}$.

Proof. Suppose that $\alpha$ is an element of $\mathbb{K}_{\infty}$ such that $|F(\alpha)| \geq \delta \widehat{P}$, where $\delta$ satisfies the hypothesis of the lemma. By Lemma 3 of [9], there exists a unique choice of $a$ and $g$ in $\mathbb{A}$ such that $g$ is monic, $(a, g)=1,1 \leq\langle g\rangle \leq \widehat{P}^{k-1}$, and $\langle g \alpha-a\rangle<\widehat{P}^{1-k}$.

Suppose that $\langle g\rangle>\widehat{P}$. It follows that $\alpha \in \mathfrak{n}$, implying that $|F(\alpha)| \ll \widehat{P}^{1-\nu}$. When $P$ is sufficiently large in terms of $k$ and $q$, one has

$$
|F(\alpha)|<\frac{1}{2} \widehat{P}^{1-\nu / 2} \leq \frac{1}{2} \delta \widehat{P}
$$

which would contradict our hypothesis on $\delta$. Hence, we may assume that $\langle g\rangle \leq \widehat{P}$. In the latter circumstance, by Lemma 4.1 of [10], we have

$$
F(\alpha) \ll \widehat{P}\left(\langle g\rangle+\widehat{P}^{k}\langle g \alpha-a\rangle\right)^{-1 / k} .
$$

Thus, there exists a positive constant $c$ such that

$$
|F(\alpha)| \leq c^{1 / k} \widehat{P}\left(\langle g\rangle+\widehat{P}^{k}\langle g \alpha-a\rangle\right)^{-1 / k} .
$$

By recalling that $\delta \widehat{P} \leq|F(\alpha)|$, we conclude that

$$
\langle g\rangle+\widehat{P}^{k}\langle g \alpha-a\rangle \leq c \delta^{-k}
$$

and the lemma follows.

We now use the hypothesis that $\lambda_{2} / \lambda_{1} \notin \mathbb{K}$ to begin our study of the product $F_{1}(\alpha) F_{2}(\alpha)$ of Weyl sums. 
Lemma 3.2. Suppose that $S$ is a fixed real number with $0<S<\widehat{\tau}^{-1}$. Then, one has

$$
\lim _{P \rightarrow \infty} \sup _{S \leq\langle\alpha\rangle<\widehat{\tau}^{-1}} \widehat{P}^{-2}\left|F_{1}(\alpha) F_{2}(\alpha)\right|=0 .
$$

Proof. Suppose that the lemma fails. We can then find a real number $\delta$ in $\left(0, q^{-1}\right)$, a sequence $\left(P_{n}\right)_{n=1}^{\infty}$ of real numbers that increases monotonically to infinity, and a sequence $\left(\alpha_{n}\right)_{n=1}^{\infty}$ of elements in $\mathbb{K}_{\infty}$ such that for all $n$, we have that $S \leq\left\langle\alpha_{n}\right\rangle<\widehat{\tau}^{-1}$ and $\left|F_{1}\left(\alpha_{n}, P_{n}\right) F_{2}\left(\alpha_{n}, P_{n}\right)\right| \geq q \delta \widehat{P}_{n}^{2}$. Since $\left|F_{i}\left(\alpha_{n}, P_{n}\right)\right| \leq q \widehat{P}_{n}$ for $i \in\{1,2\}$, one has $\left|F_{i}\left(\alpha_{n}, P_{n}\right)\right| \geq \delta \widehat{P}_{n}$ for $i \in\{1,2\}$. When $n$ is large enough, say $n \geq r$, we have that $\widehat{P}_{n}^{-\nu / 2} \leq \delta$ and that $P=P_{n}$ is sufficiently large in the context of Lemma 3.1. By Lemma 3.1, for $i \in\{1,2\}$ and $n \geq r$, there exist elements $a_{i n}$ and $g_{i n}$ in $\mathbb{A}$ such that $\left(a_{i n}, g_{i n}\right)=1$, $1 \leq\left\langle g_{\text {in }}\right\rangle \leq c \delta^{-k}$, and

$$
\left\langle g_{i n} \lambda_{i} \alpha_{n}-a_{i n}\right\rangle \leq c \delta^{-k} \widehat{P}_{n}^{-k} .
$$

It follows from the inequality for $\left\langle g_{i n}\right\rangle$ that there are only finitely many possibilities for such $g_{i n}$. For $i \in\{1,2\}$ and $n \geq r$, by noting that

$$
\left\langle a_{i n}\right\rangle \leq\left\langle g_{i n} \lambda_{i} \alpha_{n}\right\rangle+c \delta^{-k} \widehat{P}^{-k} \ll 1,
$$

we conclude that there are only finitely many choices for $a_{i n}$. Thus, there are only finitely many possibilities for the 4-tuples $\left(a_{1 n}, g_{1 n}, a_{2 n}, g_{2 n}\right)$, and some 4-tuple, say $\left(a_{1}, g_{1}, a_{2}, g_{2}\right)$, occurs infinitely often.

When $i \in\{1,2\}$ and $n \geq r$, we have

$$
\left\langle\alpha_{n}-\frac{a_{i n}}{g_{i n} \lambda_{i}}\right\rangle \leq\left\langle g_{i n} \lambda_{i}\right\rangle^{-1} c \delta^{-k} \widehat{P}_{n}^{-k} \ll \widehat{P}_{n}^{-k},
$$

and this implies that

$$
\left\langle\frac{a_{1 n}}{g_{1 n} \lambda_{1}}-\frac{a_{2 n}}{g_{2 n} \lambda_{2}}\right\rangle \ll \widehat{P}_{n}^{-k} .
$$

Since $\left(a_{1 n}, g_{1 n}, a_{2 n}, g_{2 n}\right)=\left(a_{1}, g_{1}, a_{2}, g_{2}\right)$ for infinitely many values of $n$, we conclude that

$$
\frac{a_{1}}{g_{1} \lambda_{1}}=\frac{a_{2}}{g_{2} \lambda_{2}} \text {. }
$$

If $a_{1} \neq 0$, then

$$
\frac{\lambda_{2}}{\lambda_{1}}=\frac{a_{2} g_{1}}{a_{1} g_{2}} \in \mathbb{K},
$$

which provides a contradiction. If $a_{1}=0$, by (3.1), there exists some large integer $m$ with $\left\langle\alpha_{m}\right\rangle<S$, contradicting the fact that $S \leq\left\langle\alpha_{n}\right\rangle<\widehat{\tau}^{-1}$ for all $n \in \mathbb{N}$. This completes the proof of the lemma.

We now are in a position to prove our Weyl-type estimate.

Lemma 3.3. Suppose that $S(P)$ is a function on $(0, \infty)$ that increases monotonically to infinity and satisfies $1 \leq S(P) \leq \widehat{P}$. Then, there exists a function $T(P)$ on $(0, \infty)$, depending only on $\lambda_{1}, \lambda_{2}, k, q, \tau$, and $S(P)$, that increases monotonically to infinity, satisfies $1 \leq T(P) \leq S(P)$, and satisfies the property that

$$
\sup _{S(P) \widehat{P}^{-k} \leq\langle\alpha\rangle<\widehat{\tau}^{-1}}\left|F_{1}(\alpha) F_{2}(\alpha)\right| \ll \widehat{P}^{2} T(P)^{-\nu /(2 k)} .
$$


Proof. By Lemma 3.2, for each natural number $n$, we can find a positive number $P_{n}$ such that if $P \geq P_{n}$ and $1 / n \leq\langle\alpha\rangle<\widehat{\tau}^{-1}$, then

$$
\widehat{P}^{-2}\left|F_{1}(\alpha) F_{2}(\alpha)\right| \leq \frac{1}{n}
$$

Furthermore, we can choose $\left(P_{n}\right)_{n=1}^{\infty}$ to be an increasing sequence with $S\left(P_{n}\right) \geq n$ for all $n$. Define $T(P)$ by setting

$$
T(P)= \begin{cases}n, & \text { when } P_{n} \leq P<P_{n+1}, \\ 1, & \text { when } P<P_{1} .\end{cases}
$$

If $P \geq P_{n}$ and $T(P)^{-1} \leq\langle\alpha\rangle<\widehat{\tau}^{-1}$, then

$$
\widehat{P}^{-2}\left|F_{1}(\alpha) F_{2}(\alpha)\right| \leq \frac{1}{n}
$$

This implies that

$$
\sup _{T(P)^{-1} \leq\langle\alpha\rangle<\widehat{\tau}^{-1}}\left|F_{1}(\alpha) F_{2}(\alpha)\right| \leq \widehat{P}^{2} T(P)^{-1} .
$$

Suppose now that $P$ is sufficiently large in the context of Lemma 3.1. Note that $S(P) \widehat{P}^{-k} \leq T(P)^{-1}$, and assume that

$$
S(P) \widehat{P}^{-k} \leq\langle\alpha\rangle<T(P)^{-1}
$$

and

$$
\left|F_{1}(\alpha)\right| \geq T(P)^{-\nu /(2 k)} \widehat{P}
$$

Since

$$
\widehat{P}^{-\nu / 2} \leq T(P)^{-\nu /(2 k)} \leq 1,
$$

by applying Lemma 3.1 with $\delta=T(P)^{-\nu /(2 k)}$, there exist elements $a$ and $g$ of $\mathbb{A}$ such that $(a, g)=1,1 \leq\langle g\rangle \leq c T(P)^{\nu / 2}$, and

$$
\left\langle g \lambda_{1} \alpha-a\right\rangle \leq c T(P)^{\nu / 2} \widehat{P}^{-k}
$$

Hence, by the triangle inequality,

$$
\langle a\rangle \ll\left\langle g \lambda_{1} \alpha\right\rangle+T(P)^{\nu / 2} \widehat{P}^{-k} \ll T(P)^{\nu / 2-1}+T(P)^{\nu / 2} \widehat{P}^{-k} .
$$

Since

$$
\lim _{P \rightarrow \infty}\left(T(P)^{\nu / 2-1}+T(P)^{\nu / 2} \widehat{P}^{-k}\right)=0,
$$

it follows that $a=0$ for large enough values of $P$. This implies that

$$
\langle\alpha\rangle \ll\left\langle g \lambda_{1}\right\rangle^{-1} c T(P)^{\nu / 2} \widehat{P}^{-k} \ll T(P)^{\nu / 2} \widehat{P}^{-k},
$$

and thus, for large enough values of $P$, we see that

$$
\langle\alpha\rangle<T(P) \widehat{P}^{-k} \leq S(P) \widehat{P}^{-k} .
$$

This contradicts the fact that $\langle\alpha\rangle \geq S(P) \widehat{P}^{-k}$. For $P$ sufficiently large in terms of $\lambda_{1}, \lambda_{2}$, $k, q$, and $S(P)$, we have therefore shown that whenever

$$
S(P) \widehat{P}^{-k} \leq\langle\alpha\rangle<T(P)^{-1},
$$

then

$$
\left|F_{1}(\alpha)\right|<\widehat{P} T(P)^{-\nu /(2 k)} .
$$


Hence,

$$
\sup _{S(P) \widehat{P}^{-k} \leq\langle\alpha\rangle<T(P)^{-1}}\left|F_{1}(\alpha) F_{2}(\alpha)\right| \ll \widehat{P}^{2} T(P)^{-\nu /(2 k)} .
$$

The lemma now follows by combining (3.2) with (3.3).

\section{THE MINOR ARC}

In order to complete our work on the minor arc, we first need to establish a mean value estimate for the smooth Weyl sum $f(\alpha)$.

Lemma 4.1. Suppose that $u>2 k-2$ is accessible to the exponent $k$ and that $s \geq u+5$. One has

$$
\int_{\mathbb{T}}|f(\alpha)|^{s-2} d \alpha \ll \widehat{P}^{s-2-k} .
$$

Proof. Let $v=[s / 2]-2$. By considering the underlying Diophantine equations, we note that

$$
\int_{\mathbb{T}}|f(\alpha)|^{2 v+2} d \alpha \ll \int_{\mathbb{T}}\left|F(\alpha)^{2} f(\alpha)^{2 v}\right| d \alpha
$$

Since $2 v \geq s-5 \geq u$, we may apply Lemma 6.2 of [10] to establish that

$$
\int_{\mathbb{T}}\left|F(\alpha)^{2} f(\alpha)^{2 v}\right| d \alpha \ll \widehat{P}^{2 v+2-k}
$$

and this implies that

$$
\int_{\mathbb{T}}|f(\alpha)|^{2 v+2} d \alpha \ll \widehat{P}^{2[s / 2]-2-k} .
$$

For even values of $s$, the proof of the lemma is now complete. If $s$ is odd, the lemma follows by noting that

$$
\int_{\mathbb{T}}|f(\alpha)|^{s-2} d \alpha \leq \widehat{P} \int_{\mathbb{T}}|f(\alpha)|^{2 v+2} d \alpha \ll \widehat{P}^{2[s / 2]-1-k} .
$$

We are now in a position to show that the minor arc contribution is $o\left(\widehat{P}^{s-k}\right)$, thereby confirming (2.6).

Lemma 4.2. Suppose that $u>2 k-2$ is accessible to the exponent $k$ and that $s \geq u+5$. One has

$$
\int_{\mathfrak{m}} F_{1}(\alpha) F_{2}(\alpha) f_{3}(\alpha) \cdots f_{s}(\alpha) \chi_{\tau}(\alpha) d \alpha=o\left(\widehat{P}^{s-k}\right) .
$$

Proof. By Hölder's inequality,

$$
\int_{\mathfrak{m}} F_{1}(\alpha) F_{2}(\alpha) f_{3}(\alpha) \cdots f_{s}(\alpha) \chi_{\tau}(\alpha) d \alpha \ll\left(\sup _{\alpha \in \mathfrak{m}}\left|F_{1}(\alpha) F_{2}(\alpha)\right|\right) \prod_{i=3}^{s} I_{i}^{1 /(s-2)},
$$

where

$$
I_{i}=\int_{\langle\alpha\rangle<\widehat{\tau}^{-1}}\left|f_{i}(\alpha)\right|^{s-2} d \alpha
$$


for $3 \leq i \leq s$. Note that $f(\alpha+g)=f(\alpha)$ for all $\alpha \in \mathbb{T}$ and $g \in \mathbb{A}$. By Lemma 4.1, one has

$$
\begin{aligned}
\int_{\langle\alpha\rangle<\widehat{\tau}^{-1}}|f(\alpha)|^{s-2} d \alpha & =\sum_{\substack{x \in \mathbb{A} \\
\langle x\rangle<\widehat{\tau}^{-1}}} \int_{\langle\alpha-x\rangle<1}|f(\alpha)|^{s-2} d \alpha \\
& =\sum_{\substack{x \in \mathbb{A} \\
\langle x\rangle<\widehat{\tau}^{-1}}} \int_{\mathbb{T}}|f(\alpha)|^{s-2} d \alpha \ll \widehat{P}^{s-2-k} .
\end{aligned}
$$

For $3 \leq i \leq s$, since $\left\langle\lambda_{i}\right\rangle<1$, we see that

$$
I_{i}=\left\langle\lambda_{i}\right\rangle^{-1} \int_{\langle\alpha\rangle<\left\langle\lambda_{i}\right\rangle \widehat{\tau}^{-1}}|f(\alpha)|^{s-2} d \alpha \ll \int_{\langle\alpha\rangle<\widehat{\tau}^{-1}}|f(\alpha)|^{s-2} d \alpha \ll \widehat{P}^{s-2-k} .
$$

By applying Lemma 3.3 with $S(P)=S_{1}(P)$, we obtain the bound

$$
\sup _{\alpha \in \mathfrak{m}}\left|F_{1}(\alpha) F_{2}(\alpha)\right|=o\left(P^{2}\right) .
$$

The result now follows by combining (4.1), (4.2), and (4.3).

\section{THE MAJOR ARC}

We now wish to find an asymptotic for the major arc contribution. Let

$$
\mathcal{F}(\alpha)=F_{1}(\alpha) F_{2}(\alpha) f_{3}(\alpha) \cdots f_{s}(\alpha)
$$

and

$$
\mathcal{G}(\alpha)=F_{1}(\alpha) \cdots F_{s}(\alpha)
$$

Since $S_{1}(P) \widehat{P}^{-k} \leq \widehat{\tau}^{-1}$, one has

$$
\int_{\mathfrak{M}} \mathcal{F}(\alpha) \chi_{\tau}(\alpha) d \alpha=\hat{\tau} \int_{\mathfrak{M}} \mathcal{F}(\alpha) d \alpha
$$

We first wish to compare this integral to the singular integral

$$
J_{s, k}=\int_{\langle\alpha\rangle<\widehat{P}^{1-k}} \mathcal{G}(\alpha) d \alpha
$$

To do this, let $\rho(u)$ denote the Dickman function, which is defined as the unique continuous function on $[0, \infty)$ that satisfies the differential-difference equation $u \rho^{\prime}(u)=-\rho(u-1)$ $(u>1)$ with the initial condition $\rho(u)=1(0 \leq u \leq 1)$.

Lemma 5.1. Suppose that $s \geq k+1$. One has

$$
\int_{\mathfrak{M}} \mathcal{F}(\alpha) d \alpha-\rho(P / R)^{s-2} J_{s, k} \ll \widehat{P}^{s-k}(\log \widehat{P})^{-1 /(8 k)} .
$$

Proof. Let $P$ be large enough so that $P \geq 1$ and

$$
2 P / \log (2 P)<R=\eta P<P-\log (P) .
$$

For $3 \leq i \leq s$, we deduce from Lemma 4.3 of [10] that

$$
\begin{aligned}
f_{i}(\alpha)-\rho(P / R) F_{i}(\alpha) & \ll \widehat{P}(\log \widehat{P})^{-1 / 2}\left(1+\widehat{P}^{k}\left\langle\lambda_{i} \alpha\right\rangle\right) \\
& \ll \widehat{P}(\log \widehat{P})^{-3 / 8}
\end{aligned}
$$


for $\alpha \in \mathfrak{M}$. Hence,

$$
\mathcal{F}(\alpha)-\rho(P / R)^{s-2} \mathcal{G}(\alpha) \ll \widehat{P}^{s}(\log \widehat{P})^{-3 / 8}
$$

for $\alpha \in \mathfrak{M}$. Furthermore, by noting that the measure of $\mathfrak{M}$ is $O\left(\widehat{P}^{-k}(\log \widehat{P})^{1 / 8}\right)$, one has

$$
\int_{\mathfrak{M}} \mathcal{F}(\alpha) d \alpha-\rho(P / R)^{s-2} \int_{\mathfrak{M}} \mathcal{G}(\alpha) d \alpha \ll \widehat{P}^{s-k}(\log \widehat{P})^{-1 / 4}
$$

By Lemma 4.1 of [10], whenever $1 \leq i \leq s$ and $\langle\alpha\rangle \leq \widehat{P}^{1-k}$, we have the bound

$$
F_{i}(\alpha) \ll \widehat{P}\left(1+\widehat{P}^{k}\left\langle\lambda_{i} \alpha\right\rangle\right)^{-1 / k} \ll \widehat{P}\left(1+\widehat{P}^{k}\langle\alpha\rangle\right)^{-1 / k} .
$$

Therefore,

$$
\int_{\mathfrak{M}} \mathcal{G}(\alpha) d \alpha-J_{s, k} \ll \widehat{P}^{s} \int_{\mathfrak{T}}\left(1+\widehat{P}^{k}\langle\alpha\rangle\right)^{-s / k} d \alpha,
$$

where $\mathfrak{T}=\left\{\alpha \in \mathbb{K}_{\infty}: S_{1}(P) \widehat{P}^{-k} \leq\langle\alpha\rangle\right\}$. Let $V=\log _{q}\left(S_{1}(P)\right)$. Since the measure of the set of points $\alpha$ in $\mathbb{T}$ with $\langle\alpha\rangle=q^{m}$ is less than $q^{m+1}$, we deduce that

$$
\begin{aligned}
\int_{\mathfrak{M}} \mathcal{G}(\alpha) d \alpha-J_{s, k} & \ll \widehat{P}^{s} \sum_{V-k P \leq m} q^{m+1}\left(1+q^{k P+m}\right)^{-s / k} \\
& \ll \widehat{P}^{s-k} \widehat{V}^{1-s / k} \ll \widehat{P}^{s-k}(\log \widehat{P})^{-1 /(8 k)} .
\end{aligned}
$$

The lemma now follows from (5.1).

Since $0<\eta<1$ and $R=\eta P$, we have $\rho(P / R) \gg 1$. Thus, we are left to show that $J_{s, k} \gg \widehat{P}^{s-k}$ in order to get an asymptotic lower bound of the desired form for the major arc contribution. To do this, we use ingredients from the proof of Lemma 16 in [9].

Lemma 5.2. Let $s \geq k+1$, and suppose that the equation

$$
\lambda_{1} z_{1}^{k}+\cdots+\lambda_{s} z_{s}^{k}=0
$$

has a non-trivial solution $\mathbf{z}$ in $\mathbb{K}_{\infty}^{s}$. For sufficiently large values of $P$, one has $J_{s, k} \gg \widehat{P}^{s-k}$. Proof. By Lemma 1 of [9],

$$
J_{s, k}=\int_{\langle\alpha\rangle<\widehat{P}^{1-k}} F_{1}(\alpha) \cdots F_{s}(\alpha) d \alpha=\widehat{P}^{1-k} W
$$

where $W$ denotes the number of $s$-tuples $\left(x_{1}, x_{2}, \ldots, x_{s}\right)$ in $\mathbb{A}^{s}$ with

$$
\left\langle\lambda_{1} x_{1}^{k}+\cdots+\lambda_{s} x_{s}^{k}\right\rangle<\widehat{P}^{k-1}
$$

and $\left\langle x_{i}\right\rangle \leq \widehat{P}$ for $1 \leq i \leq s$.

Choose $r$ such that $\left\langle\lambda_{r} z_{r}^{k}\right\rangle$ is maximal. Let $d=\operatorname{ord} \lambda_{r}$ and $w=\operatorname{lead}\left(\lambda_{r}\right)$. For $1 \leq i \leq s$, we define $a_{i}$ by

For $1 \leq i \leq s$, let

$$
a_{i}= \begin{cases}\operatorname{lead}\left(z_{i}\right), & \text { when }\left\langle\lambda_{i} z_{i}^{k}\right\rangle=\left\langle\lambda_{r} z_{r}^{k}\right\rangle, \\ 0, & \text { otherwise }\end{cases}
$$

$$
m_{i}=\left[\frac{d-\operatorname{ord} \lambda_{i}+k \operatorname{ord} z_{r}}{k}\right]
$$


and let $n=[P]-\max _{1 \leq i \leq s} m_{i}$. Suppose that $P$ is large enough so that $n+m_{i}>0$ for $1 \leq i \leq s$. For $1 \leq i \leq s$, let $x_{i}$ be an element of $\mathbb{A}$ with $x_{i}=a_{i} t^{n+m_{i}}+y_{i}$, where $y_{i} \in \mathbb{A}$ and ord $y_{i}<n+m_{i}$. Let

$$
x_{r}=a_{r} t^{n+m_{r}}+b_{n+m_{r}-1} t^{n+m_{r}-1}+\cdots+b_{0},
$$

where each $b_{i}$ is an element of $\mathbb{F}_{q}$, and define the coefficients $c_{l} \in \mathbb{F}_{q}$ via the relation

$$
\lambda_{1} x_{1}^{k}+\cdots+\lambda_{s} x_{s}^{k}=\sum_{l=-\infty}^{\infty} c_{l} t^{l} .
$$

The inequality (5.3) is satisfied when $c_{l}=0$ for all $l \geq(k-1) P$. For $1 \leq i \leq s$, observe that

$$
\operatorname{ord} \lambda_{i}+k\left(n+m_{i}\right) \leq d+k\left(n+m_{r}\right)
$$

with equality holding whenever $\left\langle\lambda_{i} z_{i}^{k}\right\rangle=\left\langle\lambda_{r} z_{r}^{k}\right\rangle$. Thus, the coefficient $c_{l}=0$ for all $l>d+k\left(n+m_{r}\right)$. Furthermore, our choice of $\left(a_{1}, \ldots, a_{s}\right)$ guarantees that $c_{d+k\left(n+m_{r}\right)}=0$. When

$$
d+(k-1)\left(n+m_{r}\right) \leq l<d+k\left(n+m_{r}\right),
$$

one has

$$
c_{l}=k w a_{r}^{k-1} b_{l-d-(k-1)\left(n+m_{r}\right)}+h_{l},
$$

where $h_{l}$ is an element of $\mathbb{F}_{q}$ depending at most on $\boldsymbol{\lambda}$, a, $b_{i}$ with

$$
i>l-d-(k-1)\left(n+m_{r}\right),
$$

and $y_{j}$ with $j \neq r$.

Let $y_{j}$ be arbitrarily selected for each $j \neq r$. Since $k w a_{r}^{k-1} \neq 0$, we can choose $b_{n+m_{r}-1}$ so that $c_{d+k\left(n+m_{r}\right)-1}=0$. Similarly, we can now choose $b_{n+m_{r}-2}$ so that $c_{d+k\left(n+m_{r}\right)-2}=0$. Continuing in this manner, it is possible to choose $x_{r}$ in such a way that $c_{l}=0$ for all

$$
l \geq d+(k-1)\left(n+m_{r}\right) .
$$

Since $d$ is negative, one has

$$
d+(k-1)\left(n+m_{r}\right)<(k-1) P,
$$

and it follows that $(5.3)$ holds for $\left(x_{1}, \ldots, x_{s}\right)$. Since $y_{j}$ was arbitrarily selected for $j \neq r$, for sufficiently large values of $P$, it follows that $W \gg \widehat{P}^{s-1}$, and from (5.2), we conclude that $J_{s, k} \gg \widehat{P}^{s-k}$.

By combining Lemmas 5.1 and 5.2, we obtain the following result, which confirms (2.7).

Lemma 5.3. Let $s \geq k+1$, and suppose that the equation

$$
\lambda_{1} z_{1}^{k}+\cdots+\lambda_{s} z_{s}^{k}=0
$$

has a non-trivial solution $\mathbf{z}$ in $\mathbb{K}_{\infty}^{s}$. For sufficiently large values of $P$, one has

$$
\int_{\mathfrak{M}} F_{1}(\alpha) F_{2}(\alpha) f_{3}(\alpha) \cdots f_{s}(\alpha) \chi_{\tau}(\alpha) d \alpha \gg \widehat{P}^{s-k} .
$$

By combining Lemmas 4.2 and 5.3, we have now established Theorem 2.1. 
6. The solvability of $\lambda_{1} z_{1}^{k}+\cdots+\lambda_{s} z_{s}^{k}=0$ IN $\mathbb{K}_{\infty}$

Let $\psi(q, k)$ denote the minimum integer such that for all $n>\psi(q, k)$ and any choice of $a_{1}, \ldots, a_{n} \in \mathbb{F}_{q}$, the equation $a_{1} y_{1}^{k}+\cdots+a_{n} y_{n}^{k}=0$ has a non-zero solution $\mathbf{y} \in \mathbb{F}_{q}^{n}$. We now use the function $\psi(q, k)$ to discuss the solvability of $\lambda_{1} z_{1}^{k}+\cdots+\lambda_{s} z_{s}^{k}=0$ in $\mathbb{K}_{\infty}$, which is a necessary hypothesis in Theorems 1.1 and 2.1.

Lemma 6.1. Let $\lambda_{1}, \ldots, \lambda_{s}$ be non-zero elements of $\mathbb{K}_{\infty}$. Whenever $\operatorname{char}\left(\mathbb{F}_{q}\right) \nmid k$ and $s>$ $k \psi(q, k)$, there exists a non-trivial solution $\mathbf{z} \in \mathbb{K}_{\infty}^{s}$ of the equation $\lambda_{1} z_{1}^{k}+\cdots+\lambda_{s} z_{s}^{k}=0$.

Proof. Suppose that $s>k \psi(q, k)$. Note that for any $l_{1}, \ldots, l_{s} \in \mathbb{Z}$, we have

$$
\lambda_{1} z_{1}^{k}+\cdots+\lambda_{s} z_{s}^{k}=\left(\lambda_{1} t^{-k l_{1}}\right)\left(z_{1} t^{l_{1}}\right)^{k}+\cdots+\left(\lambda_{s} t^{-k l_{s}}\right)\left(z_{s} t^{l_{s}}\right)^{k} .
$$

Hence, without loss of generality, we may assume that $0 \leq$ ord $\lambda_{i}<k$ for each $1 \leq i \leq s$, and we can find an integer $w$ with $0 \leq w<k$ such that ord $\lambda_{i}=w$ for at least $\lceil s / k\rceil$ distinct choices of $i$ with $1 \leq i \leq s$. By multiplying the equation $\lambda_{1} z_{1}^{k}+\cdots+\lambda_{s} z_{s}^{k}=0$ by $t^{-w}$, using (6.1) if necessary, and rearranging the indices if required, there is no loss of generality in supposing that ord $\lambda_{i}=0(1 \leq i \leq n)$ and ord $\lambda_{j}<0(n<j \leq s)$, where $n \geq s / k>\psi(q, k)$. Therefore, there exist elements $y_{1}, \ldots, y_{n} \in \mathbb{F}_{q}$, not all zero, such that

$$
\operatorname{lead}\left(\lambda_{1}\right) y_{1}^{k}+\cdots+\operatorname{lead}\left(\lambda_{n}\right) y_{n}^{k}=0 .
$$

By reordering the indices if necessary, we may assume that $y_{1} \neq 0$. Let $z_{i}=y_{i}(2 \leq i \leq n)$ and $z_{j}=0(n<j \leq s)$. Consider the function

$$
f(z)=\lambda_{1} z^{k}+\lambda_{2} z_{2}^{k}+\lambda_{3} z_{3}^{k}+\cdots+\lambda_{s} z_{s}^{k} .
$$

Since ord $f\left(y_{1}\right)<0$ and ord $f^{\prime}\left(y_{1}\right)=\operatorname{ord}\left(k \lambda_{1} y_{1}^{k-1}\right)=0$, an application of Hensel's lemma (see [3, Theorem 7.3]) for $\mathbb{F}_{q}[[1 / t]]$, the ring of formal power series in $t^{-1}$ with coefficients in $\mathbb{F}_{q}$, implies that there exists an element $z_{1} \in \mathbb{F}_{q}[[1 / t]] \subset \mathbb{K}_{\infty}$ such that $\operatorname{ord}\left(z_{1}-y_{1}\right)<0$ and $f\left(z_{1}\right)=0$. The lemma now follows.

By Chevalley's theorem (see Theorem 1 of Section 10.2 in [8]), we see that $\psi(q, k) \leq k$. When $q>k^{4}$, it follows from the work of Weil (see [13]) that $\psi(q, k) \leq 2$. Furthermore, when $(k, q-1)=1$, the mapping $x \mapsto x^{k}$ from $\mathbb{F}_{q}$ to $\mathbb{F}_{q}$ is a bijection, implying that $\psi(q, k)=1$. We summarize the results of this section in the following lemma.

Proposition 6.1. Suppose that $\operatorname{char}\left(\mathbb{F}_{q}\right) \nmid k$, and let $\lambda_{1}, \ldots, \lambda_{s}$ be non-zero elements of $\mathbb{K}_{\infty}$. The equation $\lambda_{1} z_{1}^{k}+\cdots+\lambda_{s} z_{s}^{k}=0$ has a non-trivial solution $\mathbf{z} \in \mathbb{K}_{\infty}^{s}$ whenever one of the following three conditions are met:

(1) $s \geq k^{2}+1$,

(2) $q>k^{4}$ and $s \geq 2 k+1$,

(3) $(k, q-1)=1$ and $s \geq k+1$.

\section{REFERENCES}

[1] V. Bentkus and F. Götze, Lattice point problems and distribution of values of quadratic forms, Ann. of Math. 150 (1999), 977-1027. 
[2] H. Davenport and H. Heilbronn, On indefinite quadratic forms in five variables, J. London Math. Soc. 21 (1946), 185-193.

[3] D. Eisenbud, Commutative algebra with a view toward algebraic geometry, Springer, New York, 1995.

[4] É. Fouvry and G. Tenenbaum, Entiers sans grand facteur premier en progressions arithmetiques, Proc. London Math. Soc. 63 (1991), 449-494.

[5] D. E. Freeman, Asymptotic lower bounds and formulas for Diophantine inequalities, Number Theory for the Millennium, Vol. 2 (Urbana, IL, 2000), A. K. Peters, Natick, MA, 2002, 57-74.

[6] D. E. Freeman, Asymptotic lower bounds for Diophantine inequalities, Mathematika 47 (2000), 127159.

[7] C.-N. Hsu, Diophantine inequalities for the non-Archimedean line $\mathbb{F}_{q}((1 / T))$, Acta Arith. 97 (2001), 253-267.

[8] K. Ireland and M. Rosen, A classical introduction to modern number theory, 2nd ed., Springer, New York, 1990.

[9] R. M. Kubota, Waring's problem for $\mathbb{F}_{q}[x]$, Dissertationes Math. (Rozprawy Mat.) 117 (1974), 60pp.

[10] Y.-R. Liu and T. D. Wooley, Waring's problem in function fields (submitted).

[11] Y.-R. Liu and T. D. Wooley, Vinogradov's mean value theorem in function fields (in preparation).

[12] R. C. Vaughan and T. D. Wooley, On Waring's problem: some refinements, Proc. London Math. Soc. 63 (1991), 35-68.

[13] A. Weil, Numbers of solutions of equations in finite fields, Bull. Amer. Math. Soc. 55 (1949), 497-508.

[14] T. D. Wooley, On Diophantine inequalities: Freeman's asymptotic formula, Bonner Math. Schriften 360 (2003), Article 30, 32pp.

School of Mathematics, Institute for Advanced Study, 1 Einstein Drive, Princeton, NJ 08540

E-mail address: craigvspencer@gmail.com 